\title{
A copper(II)-selective chelator ameliorates diabetes-evoked renal fibrosis and albuminuria, and suppresses pathogenic TGF- $\beta$ activation in the kidneys of rats used as a model of diabetes
}

\author{
D. Gong $\cdot$ J. Lu $\cdot$ X. Chen $\cdot$ S. Reddy $\cdot$ D. J. Crossman • \\ S. Glyn-Jones $\cdot$ Y.-S. Choong $\cdot$ J. Kennedy $\cdot$ B. Barry $•$ \\ S. Zhang • Y.-K. Chan $•$ K. Ruggiero • A. R. J. Phillips • \\ G. J. S. Cooper
}

Received: 8 February 2008 / Accepted: 21 May 2008 / Published online: 18 July 2008

(C) Springer-Verlag 2008

\begin{abstract}
Aims/hypothesis The selective $\mathrm{Cu}^{\mathrm{II}}$ chelator triethylenetetramine (TETA) extracts systemic $\mathrm{Cu}^{\mathrm{II}}$ into the urine of diabetic humans and rats as a model of diabetes, and in the process also normalises hallmarks of diabetic heart disease. However, the role of $\mathrm{Cu}$ and its response to TETA
\end{abstract}

This manuscript is dedicated to the memory of D. J. Scottinspirational diabetologist, teacher and humanist.

Electronic supplementary material The online version of this article (doi:10.1007/s00125-008-1088-7) contains supplementary material, which is available to authorised users.

D. Gong $\cdot$ J. Lu $\cdot$ X. Chen $\cdot$ S. Reddy $\cdot$ D. J. Crossman •

S. Glyn-Jones $\cdot$ Y.-S. Choong $\cdot$ S. Zhang $\cdot$ Y.-K. Chan $\cdot$

K. Ruggiero $\cdot$ A. R. J. Phillips $\cdot$ G. J. S. Cooper

School of Biological Sciences, Faculty of Science,

University of Auckland,

Private Bag 92019,

Auckland 1142, New Zealand

\section{S. Zhang $\cdot$ A. R. J. Phillips $\cdot$ G. J. S. Cooper}

The Maurice Wilkins Centre for Molecular BioDiscovery, University of Auckland,

Auckland, New Zealand

\section{G. J. S. Cooper $(\bowtie)$}

Medical Research Council Immunochemistry Unit, Department of Biochemistry, University of Oxford, Oxford, UK

e-mail: g.cooper@auckland.ac.nz

J. Kennedy $\cdot$ B. Barry

National Isotope Centre, GNS Science,

Gracefield,

Wellington, New Zealand in animals with diabetic nephropathy were previously unknown. Here, we report the effects of TETA treatment on $\mathrm{Cu}$ and other essential elements, as well as on indices of renal injury and known pathogenic molecular processes, in kidneys from a rat model of diabetes.

Methods Rats at 8 weeks after streptozotocin-induction of diabetes were treated with oral TETA $(34 \mathrm{mg} / \mathrm{day}$ in drinking water) for a further 8 weeks and then compared with untreated diabetic control animals.

Results Renal tissue $\mathrm{Cu}$ was substantively elevated by diabetes and normalised by TETA, which also suppressed whole-kidney and glomerular hypertrophy without lowering blood glucose. The urinary albumin:creatinine ratio was significantly elevated in the rat model of diabetes but lowered by TETA. Total collagen was also elevated in diabetic kidneys and significantly improved by TETA. Furthermore, renal cortex levels of TGF- $\beta 1$, MAD homologue (SMAD) 4, phosphorylated SMAD2, fibronectin-1, collagen-III, collagen-IV, plasminogen activator inhibitor-1 and semicarbazide-sensitive amine oxidase all tended to be elevated in diabetes and normalised by TETA.

Conclusions/interpretation Dysregulation of renal $\mathrm{Cu}$ homeostasis may be a key event eliciting development of diabetic nephropathy. Selective $\mathrm{Cu}^{\mathrm{II}}$ chelation can protect against pathogenic mechanisms that lead to or cause diabetic nephropathy and might be clinically useful in the treatment of early-stage diabetic kidney disease.

Keywords Albuminuria - Diabetic nephropathy - Divalent copper - Extracellular matrix - Glomerular hypertrophy · Semicarbazide-sensitive amine oxidase - SSAO - TGF- $\beta$. Triethylenetetramine 


$\begin{array}{ll}\text { Abbreviations } \\ \text { Ccr } & \text { creatinine clearance } \\ \text { ECM } & \text { extracellular matrix } \\ \text { PAI } & \text { plasminogen activator inhibitor } \\ \text { PAS } & \text { periodic acid-Schiff } \\ \text { PIXE } & \text { particle-induced x-ray emission spectroscopy } \\ \text { pSMAD2 } & \text { phosphorylated SMAD2 } \\ \text { SMAD } & \text { MAD homologue } \\ \text { SOD } & \text { superoxide dismutase } \\ \text { SPARC } & \text { secreted protein acidic and rich in cysteine } \\ \text { SSAO } & \text { semicarbazide-sensitive amine oxidase } \\ \text { TETA } & \text { triethylenetetramine } \\ \text { UACR } & \text { urinary albumin:creatinine ratio }\end{array}$

\section{Introduction}

Diabetic nephropathy is the most common disorder leading to end-stage renal disease worldwide. Although adequate control of blood glucose may slow the rate of its progression, many diabetic patients find it difficult to achieve strict glycaemic control in the longer term, due at least in part to limitations of available therapeutic approaches. Despite the availability of treatments that lower elevated levels of blood glucose and/or blood pressure, many diabetic patients are still prone to developing kidney failure that no currently available therapies can reverse [1]. It is thus appropriate to search for new therapeutic approaches to the treatment of diabetic nephropathy, based on novel mechanisms of action.

Transforming growth factor- $\beta$ is a key mediator of tissue extracellular matrix (ECM) build-up in experimental and human renal disease. It reportedly stimulated ECM protein accumulation in diabetic tissues by upregulating production of ECM proteins and/or downregulating production of ECM-degrading enzymes [2]. Increased renal levels of TGF- $\beta 1$ have been reported in both experimental and human diabetes [3]. Furthermore, TGF- $\beta 1$ elicits synthesis of ECM components including collagens I, III and IV, and fibronectin $[4,5]$. We previously reported that the chelatable $\mathrm{Cu}^{\mathrm{II}}$ content increased in myocardium from rats used as a model of diabetes and that a highly selective $\mathrm{Cu}^{\mathrm{II}}$ chelator, triethylenetetramine (TETA), reversed the structural and functional hallmarks of diabetes-induced cardiac disease in rats and humans $[6,7]$. TETA may evoke these effects, at least in part, by suppressing the TGF- $\beta$-mediated upregulation of ECM proteins and restoring physiological extracellular superoxide dismutase (SOD)-3 [7, 8] and heparan sulphate regulation in myocardial and arterial tissues [8]. Renal $\mathrm{Cu}$ content was reportedly increased in hypertensive rats $[9,10]$ and elevated urinary $\mathrm{Cu}$ excretion has been reported in diabetic patients with nephropathy [11]. However, it was not previously known whether TETA-evoked $\mathrm{Cu}^{\text {II }}$ chelation might improve renal structure and function in diabetes, while the molecular basis for any putative actions that TETA might have in individuals with diabetic nephropathy remained to be elucidated. For example, the observation that $\mathrm{Cu}^{\mathrm{II}}$ is essential for the activity of the $\mathrm{Cu}^{\mathrm{II}}$ dependent enzyme, semicarbazide-sensitive amine oxidase (SSAO) may be relevant to TETA's actions in the ECM. The major circulating SSAO activity is amine oxidase, copper containing 3 (AOC3), encoded by the gene Aoc3. Increased circulating SSAO activity has been reported in human diabetic patients [12] and the enzyme is believed to enhance the synthesis of AGE in the macrovasculature [13]. Moreover, transgenic mice overexpressing $A O C 3$ in endothelial cells develop a form of nephropathy [14] similar to that in diabetes.

Activation of the TGF- $\beta$ pathway has been shown to result in many fibrotic diseases [15]. Treatment with $160 \mu \mathrm{g} / \mathrm{kg} \mathrm{Cu}$ reportedly increased renal tgfb mRNA levels in a fish species [16]. Tetrathiomolybdate, a potent $\mathrm{Cu}-$ chelating drug, attenuated cholestatic liver injury and fibrosis in mice, in part by inhibiting TGF- $\beta 1$ secretion [17]. However, it previously remained unknown whether TETA could suppress activation of the TGF- $\beta$ pathway in diabetic kidneys. In this study, we examined the effects of TETA on hallmarks of nephropathy in streptozotocininduced diabetic rats, a widely used model for mechanistic studies of human diabetic nephropathy [18, 19].

\section{Methods}

Animals These studies were approved by the University of Auckland Animal Ethics Committee. Male Wistar rats (220 250 g) (Jackson Laboratories, Bar Harbor, ME, USA) were rendered diabetic by a single intravenous injection of streptozotocin $(55 \mathrm{mg} / \mathrm{kg}$ body weight; Sigma-Aldrich, St Louis, MO, USA) as described [6, 8]. Body weights and blood glucose values were monitored weekly for 16 weeks. At week 9 after streptozotocin injection, the rats were randomly divided into two groups: untreated diabetic and TETAtreated diabetic. TETA disuccinate $(34 \mathrm{mg} /$ day per rat; Protemix, Auckland, New Zealand) was administered in the drinking water (18 M $\Omega$; Milli Q, Millipore, Billerica, MA, USA) to streptozotocin-induced diabetic rats for a further 8 weeks. At 16 weeks after diabetes induction, rats were anaesthetised and killed. Both kidneys were immediately excised and weighed. The left kidney was divided into two halves, one of which was sliced into small pieces, washed thoroughly in DEPC-treated phosphate-buffered saline and stored in RNAlater (Ambion, Austin, TX, USA). A portion of the washed tissue was also stored at $-80^{\circ} \mathrm{C}$ for protein analysis. The other half of each left kidney was further divided into two halves, which were either snap-frozen in liquid nitrogen or fixed in $10 \%$ (vol./vol.) formalin solution 
for histological analysis. For metal measurements, the left kidney was excised using titanium instruments.

Measurement of copper and other elements Particleinduced x-ray emission spectroscopy (PIXE) with concomitant Rutherford backscatter spectroscopy was used to measure renal concentrations of nine elements as described [20]. This quantitative technique was employed to allow for possible changes in the tissue ratios of elements between the extracellular and intracellular compartments. The calibration, measurements and limits of detection were based on the areas of the $K_{\alpha}$ X-ray peaks as assessed by the software package GUPIX Elemental and concentrations were extracted from PIXE spectra using GUPIX software (University of Guelph, ON, Canada).

Urine collection and analysis Rats were placed individually in metabolism cages and $24 \mathrm{~h}$ urine samples collected from two similarly treated cohorts at week 15 in metal-free propylene tubes. Samples were kept at $-80^{\circ} \mathrm{C}$ until assay. Urinary albumin content was determined using a kit (Rat Albumin Enzyme Immunoassay; SPI-BIO, QC, Canada) and urinary creatinine by a modified Jaffé method; urinary albumin:creatinine ratio (UACR) was calculated from these two measurements. Creatinine clearance was calculated on the basis of urinary creatinine, serum creatinine, urine volume and body weight using the following equation: $\operatorname{Ccr}\left(\mathrm{ml} \mathrm{s}^{-1}[\mathrm{~kg} \text { body weight }]^{-1}\right)=[$ urinary $\operatorname{Cr}(\mu \mathrm{mol} / 1) \times$ $[24 \mathrm{~h}$ urine volume $(\mathrm{ml})] / \operatorname{serum~} \mathrm{Cr}(\mu \mathrm{mol} / \mathrm{l})] \times[1000 /$ body weight $(\mathrm{g})] \times[1 / 86,400(\mathrm{~s})]$, where $\mathrm{Ccr}$ is creatinine clearance and $\mathrm{Cr}$ is creatinine.

Determination of glomerular area Light microscopy of periodic acid-Schiff (PAS)-stained kidney sections (6 $\mu \mathrm{m}$ thick) was used for morphometric studies. The surface area $\left(\mu \mathrm{m}^{2}\right)$ of at least 50 random glomerular sections from each animal was determined in digital images $(\times 20$ objective magnification) by a single blinded observer using the Soft Imaging System (Olympus, London, UK).

$R N A$ isolation and quantitative real-time $R T-P C R$ Total RNA was isolated from renal cortex with a kit (RNeasy Midi; Qiagen, Hilden, Germany) and used for cDNA synthesis by reverse transcription. Quantitative real-time PCR was performed using a Prism 7900 sequence detection system (Applied Biosystems, Foster City, CA, USA) and specific primers as described [8]. The levels of gene expression of the target sequence were normalised to those of $18 \mathrm{~S}$ ribosomal RNA ( $r 18 S$; Ambion) and data were analysed with sequence detector software (Prism 7900; Applied Biosystems).

Western blotting Polyclonal anti-fibronectin-1, anti-collagen IV, anti-plasminogen activator inhibitor (PAI)-1, anti-SOD-1 and anti-SSAO antibodies were from Santa Cruz Biotechnology (Santa Cruz, CA, USA), whereas polyclonal anti-phospho-MAD homologue (SMAD)2 and anti-SOD-3 antibodies were from Chemicon International (Temecula, CA, USA) and Stressgen (Victoria, BC, Canada), respectively. Total protein was isolated from left kidneys in extraction buffer (50 mmol/1 Tris- $\mathrm{HCl}, \mathrm{pH} \mathrm{7.4,} 150 \mathrm{mmol} / \mathrm{l} \mathrm{NaCl}$, $5 \mathrm{mmol} / \mathrm{l}$ EDTA, $10 \mathrm{mmol} / \mathrm{l}$ DTT and 1\% (vol./vol.) Triton $\mathrm{X}-100$ ) with the addition of a protease inhibitor cocktail (Roche, Basel, Switzerland). Protein samples were then separated by SDS-polyacrylamide gel electrophoresis and immunodetection performed with an electrochemoluminescence kit (ECL Plus; GE Healthcare, Amersham, UK).

Immunohistochemical analysis Frozen kidney blocks were sectioned transversely $(6 \mu \mathrm{m})$, fixed briefly in cold acetone and stored at $-20^{\circ} \mathrm{C}$ until analysis. Prior to immunohistochemistry, cryosections were fixed in cold acetone for $10 \mathrm{~min}$, equilibrated in phosphate-buffered saline and blocked with $10 \%$ (vol./vol.) normal donkey serum. Sections were then incubated for $18 \mathrm{~h}$ at $4{ }^{\circ} \mathrm{C}$ with rabbit anticollagen IV (1:100; Rocklands Laboratories, Gilbertsville, PA, USA). After washing, sections were incubated with donkey anti-rabbit IgG-biotin $\left(1: 100 ; 1 \mathrm{~h}, 37^{\circ} \mathrm{C}\right.$; Jackson Immunoresearch, West Grove, PA, USA) followed by reaction with streptavidin-Texas Red $\left(1: 100,1 \mathrm{~h}, 37^{\circ} \mathrm{C}\right.$; Jackson ImmunoResearch). Sections were imaged with an Axiovert S100 microscope equipped with a digital camera and Axiovision 3 software (Carl Zeiss, Oberkochen, Germany).

Determination of total collagen, TGF- $\beta 1$ and heparan sulphate Total collagen was assayed by measuring the concentration of hydroxyproline as described [21]. The level of active TGF- $\beta 1$ was determined by an immunoassay system (TGF- $\beta 1 \mathrm{E}_{\max }$; Promega). The optical density at $450 \mathrm{~nm}$ was measured on a microplate reader (Spectra Max 340; Molecular Devices, Sunnyvale, CA, USA). Tissue heparan sulphate levels were determined by an heparan sulphate ELISA kit (Seikagaku, Chuo-Ku, Tokyo, Japan) [8].

Statistics Data are expressed as the means \pm SEM and analysed by one-way ANOVA and post-hoc pairwise comparisons. In all visual material, means and SEM are presented on the original scale. Analyses were performed using SAS version 9.1 (SAS Institute, Cary, NC, USA) or Prism version 4.2 (GraphPad Software, San Diego, CA, USA), and R version 2.4.1 for the Kruskal-Wallis and Wilcoxon rank sum tests, $t$ tests and Bonferroni corrections. The method applied in each case is as specified in the figure legends. A value of $p<0.05$ was considered as statistically significant. 


\section{Results}

TETA normalised renal copper accumulation in diabetic rats We had previously reported that TETA stimulated urinary $\mathrm{Cu}$ excretion in diabetic patients $[6,7]$ and rats [6]. To characterise the interactive effect of diabetes and TETA on the regulation of renal trace metal metabolism, we measured concentrations of $\mathrm{Cu}$ and other essential trace metals in kidneys from untreated diabetic and TETA-treated diabetic rats. Diabetes significantly elevated renal content of $\mathrm{Cu}$ and $\mathrm{Zn}$ compared with non-diabetic controls (Fig. 1a, b). In contrast, we found no change in renal content of $\mathrm{Fe}$, $\mathrm{Na}, \mathrm{K}, \mathrm{Mg}, \mathrm{Ca}, \mathrm{P}$ or $\mathrm{Cl}$ between diabetic and non-diabetic rats, nor were these values modified by TETA (results not shown). TETA treatment largely restored renal $\mathrm{Cu}$ content towards that of controls (Fig. 1a), whereas it did not normalise renal $\mathrm{Zn}$ content (Fig. 1b). These results indicate that diabetes impaired renal $\mathrm{Cu}$ and $\mathrm{Zn}$ regulation, leading to a substantive build-up of both metals in the renal tissue. The upregulation of $\mathrm{Cu}$ was selectively reversed by TETA, thereby confirming its in vivo selectivity over all other physiological divalent metal ions.

TETA attenuated renal and glomerular hypertrophy in diabetic rats Hyperglycaemia persisted at equivalent levels in untreated and TETA-treated diabetic rats throughout the

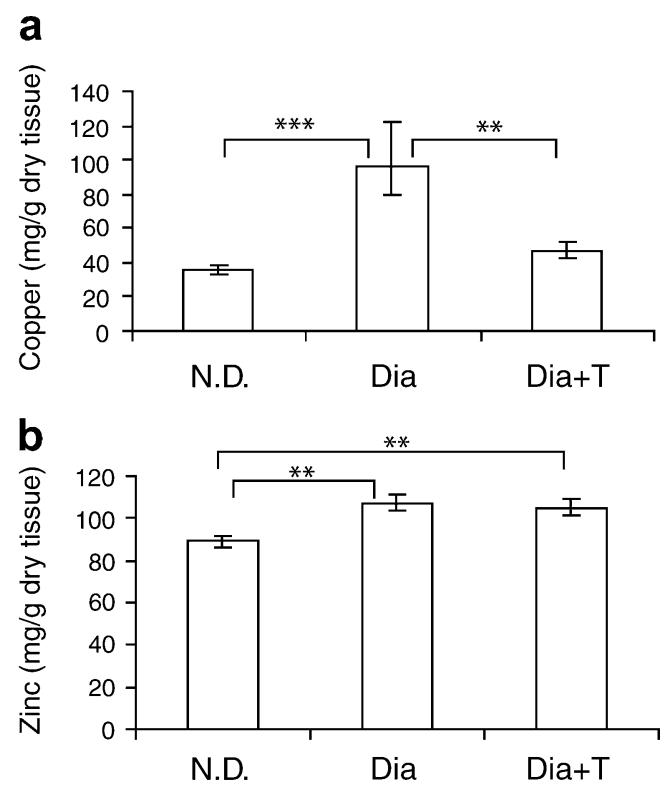

Fig. 1 Effect of TETA administration on total copper (a) and zinc (b) concentrations in the kidneys of diabetic rats. Total copper and zinc levels in whole left kidneys from non-diabetic (N.D.), streptozotocininduced diabetic (Dia) and TETA-treated diabetic (Dia+T) rats were measured by PIXE. Reciprocal transformation of data was performed prior to analysis. Data are means \pm SEM; $n=7$ per group; ${ }^{* *} p<0.01$, $* * * p<0.001$ by one-way ANOVA with post hoc Tukey's tests study period. Blood glucose levels in diabetic rats $(31.8 \pm$ $0.3 \mathrm{mmol} / 1$ at the end of week 16) were markedly increased compared with the non-diabetic controls $(4.8 \pm 0.2 \mathrm{mmol} / \mathrm{l})$, whereas no significant difference in blood glucose levels was seen between TETA-treated $(30.8 \pm 0.8 \mathrm{mmol} / \mathrm{l}$ at the end of week 16) and untreated diabetic rats, indicating that the drug did not lower glycaemia in diabetic rats. Body weight increase in diabetic rats $(72.3 \pm 19.0 \mathrm{~g}$ at end week 16) was similar to that in TETA-treated diabetic rats $(66.5 \pm 17.0 \mathrm{~g})$, but significantly less than in non-diabetic controls $(285.6 \pm 10.6 \mathrm{~g})$. This result suggests that 8 weeks of TETA treatment did not have toxic effects. A clinical trial showed TETA treatment to be safe and well tolerated by type 2 diabetic patients given $1,200 \mathrm{mg}$ trientine/day for 6 months [6]. Untreated diabetes caused renal hypertrophy characterised by significantly increased kidney weight $(1.99 \pm 0.14 \mathrm{~g})$ compared with non-diabetic controls $(1.55 \pm$ $0.05 \mathrm{~g})$, as well as elevated kidney weight:body weight ratios (Fig. 2a). Renal hypertrophy was significantly ameliorated by TETA treatment (Fig. 2a).

We next determined whether TETA-evoked amelioration of renal hypertrophy in diabetic rats was accompanied by a similar decrease in glomerular hypertrophy. Glomeruli from untreated diabetic animals tended to be larger than those from non-diabetic controls, whereas glomerular area was significantly decreased by TETA vs untreated diabetic rats (Fig. 2b,c). Collectively, these results demonstrate that TETA decreased both renal hypertrophy and glomerular hypertrophy in diabetic animals, without changing blood glucose concentrations.

TETA decreased urinary albumin excretion in diabetic rats Untreated diabetic rats exhibited a significant increase in urinary albumin excretion (expressed as UACR) when compared with non-diabetic controls. TETA significantly decreased UACR towards normal in diabetic rats (Fig. 3a). Thus, TETA significantly suppressed the development of albuminuria in diabetic rats. Urinary urea excretion rates were significantly increased in diabetic rats, consistent with diabetes-evoked hyperphagia and hyperfiltration, but were unaltered by TETA (Fig. 3b). No significant difference was observed in creatinine clearance among non-diabetic, diabetic and TETA-treated diabetic groups (Fig. 3c).

TETA normalised hydroxyproline content of diabetic kidneys To determine whether TETA could exert measurable effects on the regulation of collagen metabolism in the diabetic model of renal fibrosis, we evaluated renal fibrosis by measuring the content of hydroxyproline, a major specialised amino acid of collagen, in whole-kidney homogenates. Renal hydroxyproline levels were significantly elevated in untreated diabetic animals compared with those of non-diabetic controls, but were normalised 

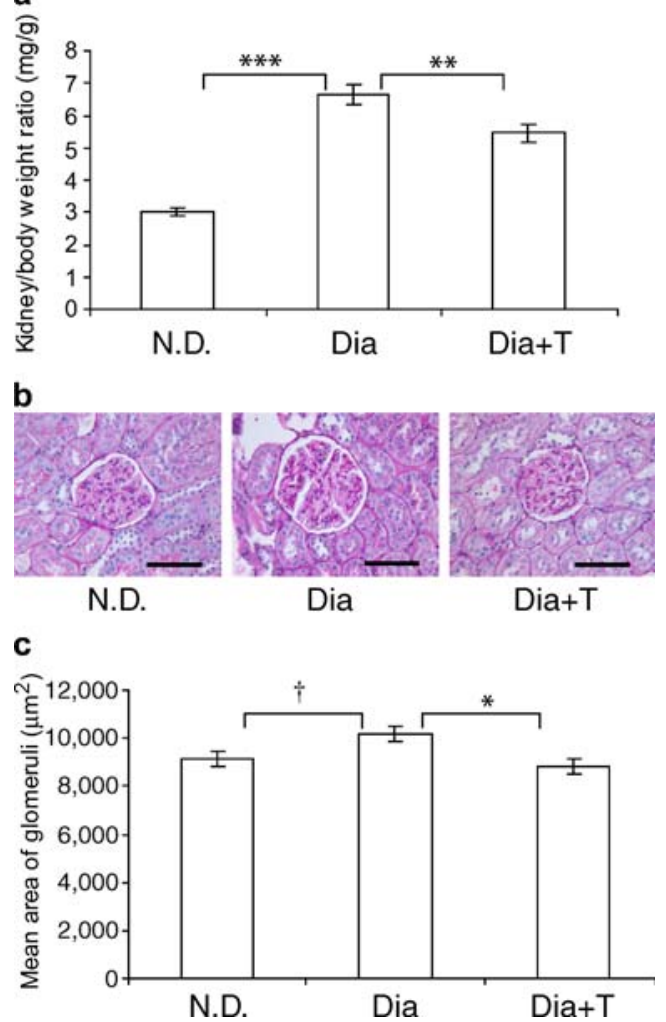

Fig. 2 Effect of TETA treatment on kidney weight:body weight ratio and glomerular hypertrophy in diabetic rats. a Kidney weight:body weight ratio in non-diabetic control (N.D., $n=6$ ), streptozotocindiabetic (Dia, $n=9)$ and TETA-treated diabetic $(\mathrm{Dia}+\mathrm{T})$ groups $(n=9)$. Data were log-transformed prior to analysis. b Representative photomicrographs of kidney sections stained with PAS from non-diabetic control, diabetic and TETA-treated diabetic groups $(n=8)$. Scale bar, $50 \mu \mathrm{m}$. c Measurement of glomerular size. Glomerular cross-sectional areas were measured from kidney sections stained with PAS using Soft Imaging System software. Bars represent means \pm SEM from at least 50 individual glomeruli in sections from each of eight individual rats in each group. ${ }^{*} p<0.05,{ }^{* *} p<0.01,{ }^{* * *} p<0.001$ and $\dagger p=0.07$ by one-way ANOVA with post hoc Tukey's tests

following TETA treatment (Fig. 4a). It has previously been shown that diabetes-evoked decreases in heparan sulphate levels both in the heart and aorta of rats were attenuated by TETA [8]. In contrast, renal heparan sulphate content was not changed in our diabetic animals and was unaffected by TETA (Fig. 4b).

TETA attenuated levels of ECM proteins in diabetic kidneys To investigate the molecular mechanism by which TETA improved kidney structure and function in diabetic rats (Figs 2 and 3), we analysed content in kidney cortex of three ECM proteins (fibronectin-1, collagen III and collagen IV), which are major components of the glomerular basement membrane. Levels of mRNAs corresponding to fibronectin-1, collagen III and collagen IV (Fnl, Col3al and Col4a3, respectively) were elevated by 1.91-, 1.42- and 1.32-fold respectively in the kidneys of 16-week diabetic rats as compared with non-diabetic control animals (Fig. 5). Furthermore, TETA treatment significantly suppressed these mRNA increases in the kidneys of diabetic rats (Fig. 5).

Western blotting also showed that protein levels of fibronectin-1 and collagen IV in the renal cortex were significantly elevated in diabetic animals, as compared with non-diabetic controls (Fig. 5b,e). The increased levels of these proteins tended to be decreased in TETA-treated diabetic rats (Fig. 5b,e). These observations were further confirmed by immunohistochemical analyses. For example, deposition of collagen IV in glomeruli and tubules was increased in the diabetic group, but TETA diminished the build-up of this protein (Fig. 5f).
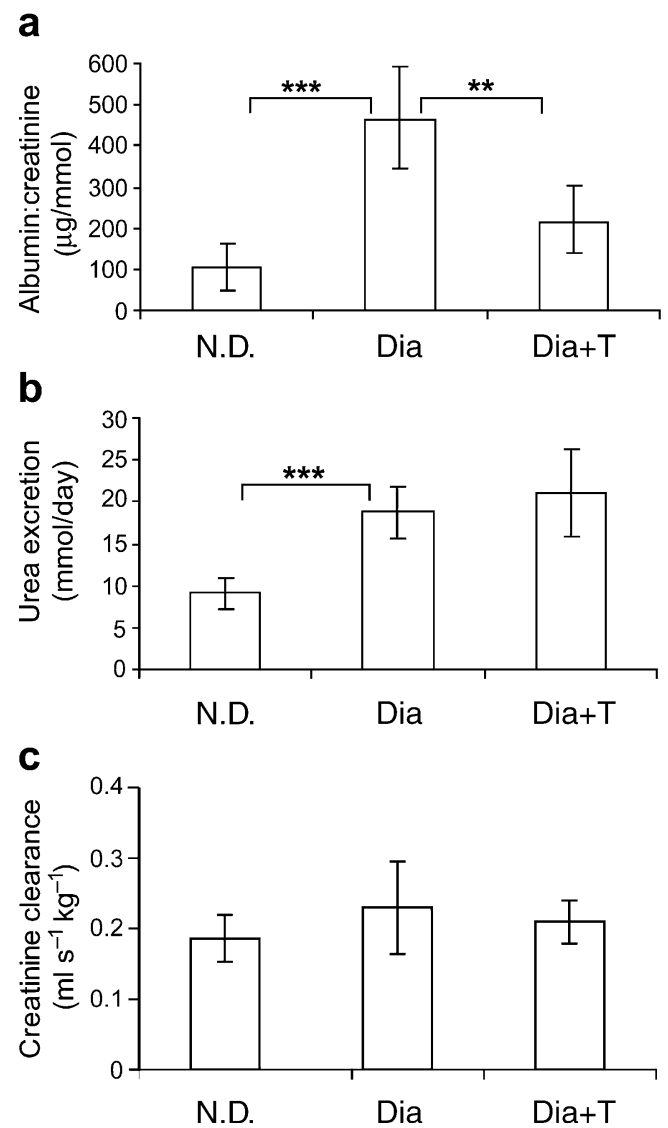

Fig. 3 Effect of TETA treatment on urine parameters. a UACR, adjusted means $\pm \mathrm{SEM}$, (b) urine urea and (c) creatinine clearance in non-diabetic (N.D., $n=6$ ), streptozotocin-induced diabetic (Dia, $n=13$ ) and TETA-treated diabetic (Dia+T, $n=13$ ) rats. TETA was administered to rats at 8 weeks after streptozotocin induction of diabetes for a further 7 weeks. A 24 h pooled urine sample was collected from each animal in a metabolism cage at week 15 (week 7 of diabetes) for each group. Square-root transformation was applied to UACR values (a) and a linear mixed-effects model, adjusting for inter-run variability, was then fitted. UACR means, adjusted for imbalances between operators and their standard errors, are presented. All data are means \pm SEM and were analysed with a linear mixed-effects model (a) and one-way ANOVA with post hoc Tukey's tests $(\mathbf{b}, \mathbf{c}) .{ }^{* *} p<$ $0.01, * * * p<0.001$ 
a

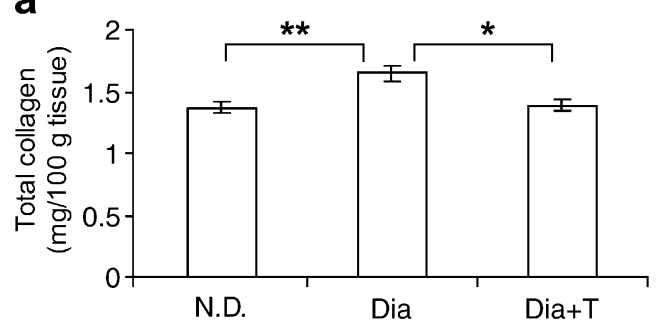

b

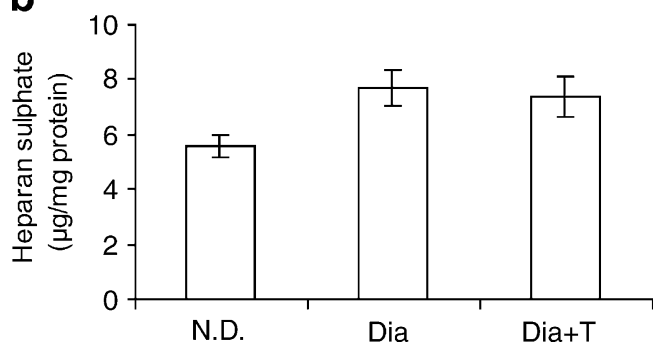

Fig. 4 Effect of TETA administration on total collagen level (a) and heparan sulphate content (b) of diabetic kidneys. TETA was administered to rats at 8 weeks after streptozotocin induction of diabetes for a further 8 weeks. Total collagen content in both kidneys was determined by measuring hydroxyproline concentration in each specimen. Dia, diabetic $(n=7)$; Dia + T, TETA-treated diabetic rats $(n=$ 7); N.D., non-diabetic $(n=5)$. Transformation of heparan sulphate data, satisfying the ANOVA requisites, was not identified and the data were consequently analysed using the Kruskal-Wallis and Wilcoxon rank sum tests with Bonferroni correction. Data are means \pm SEM and were analysed by one-way ANOVA with post hoc Tukey's tests (a) and the Kruskal-Wallis test (b). ${ }^{*} p<0.05,{ }^{*} p<0.01$

Overexpression of Tgfb1, Smad4 and Serpine1/Pail in diabetic kidneys was ameliorated by TETA To further understand the mechanism by which TETA inhibited accumulation of ECM proteins in diabetic kidneys, we analysed expression of some key downstream molecules in the TGF- $\beta$ signalling pathway. Diabetic rats exhibited elevated mRNA expression of $T g f b 1$ and Smad4 in renal cortex compared with non-diabetic animals, which was downregulated by TETA (Fig. 6a,d). TETA also suppressed the increased TGF- $\beta 1$ protein levels in diabetic kidneys, as determined by immunoblotting (Fig. 6b). ELISA analysis further showed that the increased, biologically active TGF- $\beta 1$ protein in diabetic kidneys tended to decrease following TETA treatment (Fig. 6c). In addition, western blotting showed that protein levels of phosphorylated SMAD2 (pSMAD2), a marker of TGF- $\beta$ signalling activation, were significantly increased in diabetic kidneys (Fig. 6e). Interestingly, TETA significantly inhibited phosphorylation of SMAD2 in diabetic kidneys (Fig. 6e). Taken together, these results indicate that TETA suppressed ECM expansion and renal hypertrophy, at least in part via downregulation of the TGF- $3 /$ SMAD signalling pathway. In addition, diabetic rats exhibited increased expression of Serpine1/Pail mRNA (Fig. 6f) and protein (Fig. 6g) compared with non-diabetic animals; this increase was reversed by TETA (Fig. 6f, g).

TETA ameliorated levels of SSAO in diabetic kidneys SSAO protein levels in the kidney were significantly increased in diabetic rats as compared with non-diabetic controls. However, TETA downregulated SSAO protein content in diabetic kidneys, as shown in Electronic supplementary material (ESM, Fig. 1). By contrast, no significant difference in renal protein levels of two other $\mathrm{Cu}$-containing enzymes, $\mathrm{Cu} / \mathrm{Zn}$ SOD-1 and SOD-3, could be demonstrated between diabetic and non-diabetic rats or between TETAtreated and untreated diabetic rats (ESM Fig. 1).

\section{Discussion}

To our knowledge, strategies to target $\mathrm{Cu}$ regulation for pharmacotherapy of renal fibrosis in diabetes have not previously been systematically explored. This study is the first to demonstrate marked suppression of renal fibrosis in vivo by a highly selective $\mathrm{Cu}^{\text {II }}$ chelator [6], as demonstrated both histologically (Figs 2 and 5) and by decreased renal content of hydroxyproline, collagen and fibronectinspecific biochemical markers for tissue fibrosis (Figs 4 and 5). Perhaps more significantly, TETA also suppressed diabetesevoked increases in UACR, a key measure of renal function, in diabetic rats.

We had previously reported that TETA improved hallmarks of heart disease in diabetic rats and patients with type 2 diabetes [6,7]. However, until the current study, it was not known whether TETA could exert any renoprotective effect in diabetes. To address this question, we evaluated the effect of TETA on urinary albumin excretion in diabetic rats. Urinary albumin has been suggested as a predictive indicator for prognosis of diabetic nephropathy and its elevated excretion is also said to impair renal function [22]. Furthermore, reductions in urinary albumin in diabetic nephropathy were reportedly associated with renal protection [23]. Here we show that diabetes caused significant increases in the UACR, whereas TETA suppressed this effect (Fig. 3a), demonstrating a renoprotective effect of TETA in a rat model of diabetic nephropathy. In another report, renal $\mathrm{Cu}$ levels were reportedly increased in 3 week streptozotocin diabetic rats, but elevated renal $\mathrm{Cu}$ in those animals did not affect renal function in the early stages of diabetes [24], possibly consistent with the shorter diabetic period in that study. Previous studies have also shown TETA dihydrochloride to act as a $\mathrm{Cu}$-selective chelator [25, 26]. We are interested in comparing the effects of TETA with those of other $\mathrm{Cu}$ chelators and agents shown to attenuate or prevent indices of diabetic nephropathy. Here, the increased blood glucose in diabetic rats was not altered 
a
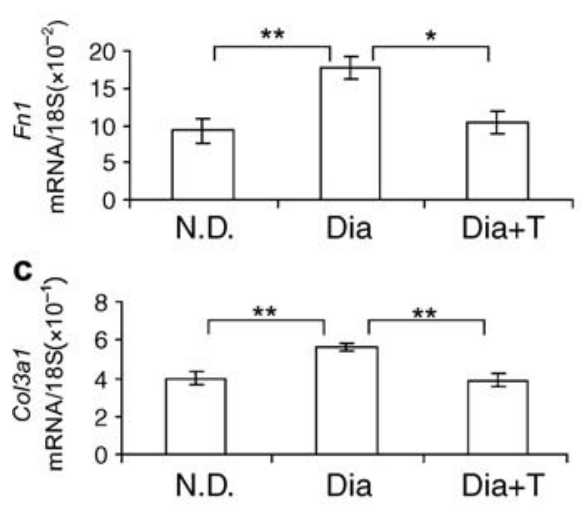

e

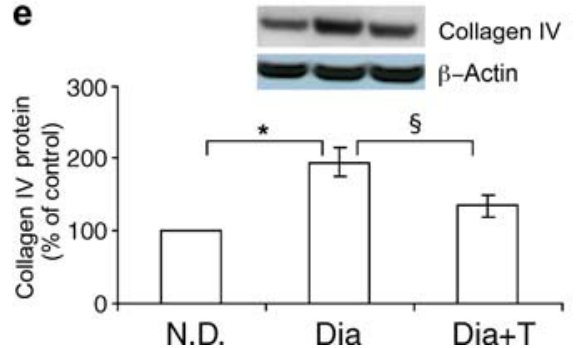

Fig. 5 TETA treatment normalised mRNA and protein levels of ECM components in diabetic kidneys. a Fn1 mRNA level. b Representative western analysis with blot and relative measurement of content of fibronectin-1. c Col3al mRNA expression. d Col4a3 mRNA expression. e Representative western analysis with blot and relative measurement of levels of collagen IV in rat kidneys. f Immunohistochemical staining of collagen IV in representative kidney sections $(n=$ 5/group). Dia, diabetic; Dia+T, TETA-treated diabetic; N.D., non diabetic. Phosphate-buffered saline was used as a negative control. Sections were counterstained with streptavidin-Texas Red (1:100). TETA was administered to rats at 8 weeks after streptozotocin induction of diabetes for a further 8 weeks. RNA was isolated from left kidneys and mRNA levels were determined by real-time quantitative PCR. Col4a3 and Smad4 mRNA expression data were $\log$-transformed prior to analysis. A transformation of Col3al data

by TETA, indicating that its renoprotective effect was independent of any possible hypoglycaemic action. It remains unknown whether TETA prevents renal $\mathrm{Cu}$ accumulation by sequestering the metal in the serum and thus preventing $\mathrm{Cu}$ entry into the renal cells or by stimulating its excretion from renal cells or by both mechanisms.

Although the pathogenesis of diabetic kidney disease is multi-factorial, tissue fibrosis is a key pathological hallmark of this disease, in which build-up of ECM proteins is a key pathogenic process. Hyperglycaemia in diabetes is known to increase the synthesis of ECM components in the kidneys. In this study, the increase in total collagen and content of the major ECM components, fibronectin-1, collagen III and collagen IV, occurred in experimental diabetes (Figs 4 and 5). Our recent transcriptomic analysis has also identified increased content of mRNAs corres-
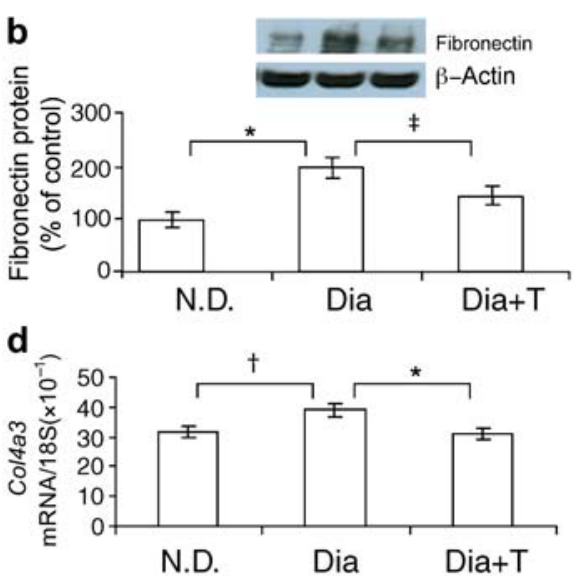

f

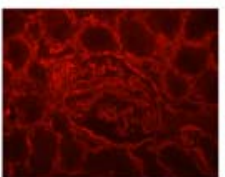

N.D.

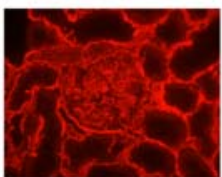

Dia

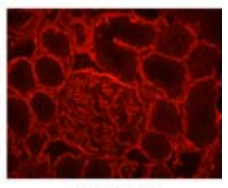

Dia+T that satisfied ANOVA requirements was not identified and these were consequently analysed using the Kruskal-Wallis and Wilcoxon rank sum test with Bonferroni correction. Collagen IV protein content data required normalisation to non-diabetic control values, so these were analysed by two-sample $t$ tests for the comparison of diabetic and TETA-treated diabetic rats; one sample $t$ tests were then used to compare the mean value from diabetic and TETA-treated diabetic groups to the mean of the control group. $p$ values were adjusted using the Bonferroni correction. The band intensity of fibronectin-1 or collagen IV in non-diabetic controls was designated as $100 \%$. $\beta$-Actin was used as a loading control. Data represent means \pm SEM $(n=5 /$ group) and were analysed by one-way ANOVA with post hoc Tukey's tests, and as described above. ${ }^{*} p<0.05,{ }^{*} p<0.01, \dagger p=0.07, \ddagger p=0.08$, $\S p=0.09$

ponding to fibronectin-1 and collagen IV, respectively, in the heart and aorta of diabetic rats [8]. Here, we found that TETA normalised diabetes-induced elevations in total collagen, fibronectin-1, collagen III and collagen IV proteins (Figs 4 and 5). Our recent proteomic analysis showed that diabetes-induced upregulation of the major receptor, $\beta_{1}$ integrin, of another ECM protein, laminin, was attenuated by TETA in the diabetic aorta (X. Chen, D. Gong, A. R. J. Phillips and G. J. S. Cooper, unpublished observations), consistent with our previous findings in the diabetic heart [6]. It remains unknown whether TETA modifies content of laminin and its receptors in the diabetic kidney.

TGF- $\beta 1$ reportedly induces mesangial expansion and glomerular basement membrane thickening through ECM protein build-up in diabetic kidneys [27]. Here, we have shown that increased TGF- $\beta 1$ levels in diabetic kidneys 
Fig. 6 Suppression of TGF- $\beta 1$, SMAD4 and PAI-1 in the kidneys of streptozotocindiabetic rats by TETA treatment. a $T g f b 1$ mRNA expression. b A representative western analysis with blot of TGF- $\beta 1$ protein and its relative levels as a bar graph. c TGF- $\beta 1$ protein levels determined by ELISA. d Smad4 mRNA expression. e A representative western analysis with blot of pSMAD2 and its relative levels as a bar graph. f Serpine 1/ Pail mRNA expression. g A representative western analysis with blot of SERPINE1/PAI1 and its relative levels as a bar graph. Dia, diabetic; Dia+T, TETA-treated diabetic; N.D., non-diabetic. TETA was administered to diabetic rats for a further 8 weeks at 8 weeks after streptozotocin induction of diabetes. Total RNA was isolated from kidneys and the mRNA expression level was quantified by quantitative real-time PCR. $\beta$-Actin was used as a loading control. The band intensity of either TGF- $\beta 1$, pSMAD2 or PAI-1 protein in the nondiabetic control was designated as $100 \%$. Each value represents means $\pm \operatorname{SEM}$ ( $n=5$ per group). Data were analysed by one-way ANOVA with post hoc Tukey's tests. ${ }^{*} p<0.05,{ }^{* *} p<0.01, \dagger p=$ 0.06, $₫ p=0.09$ a

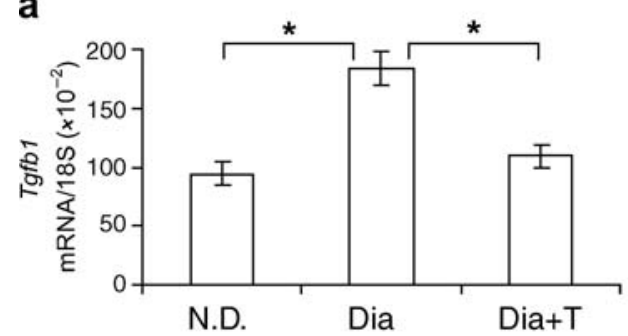

C
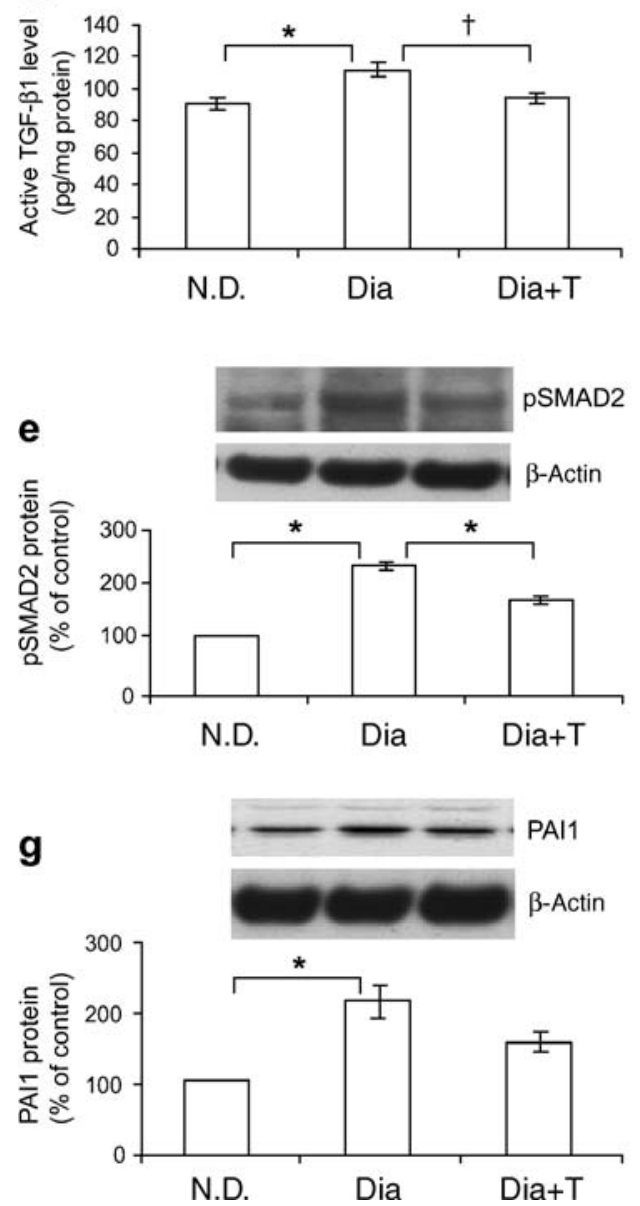

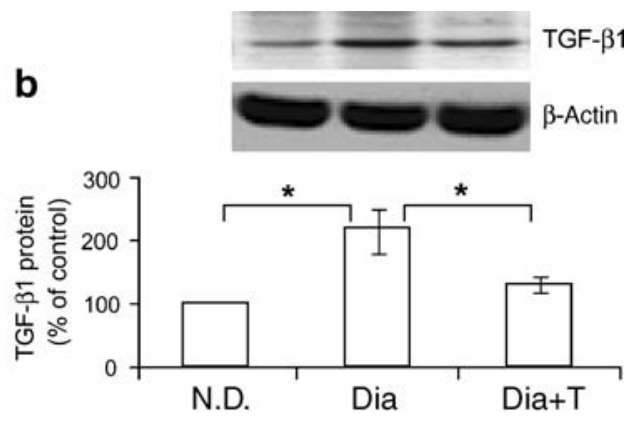

d
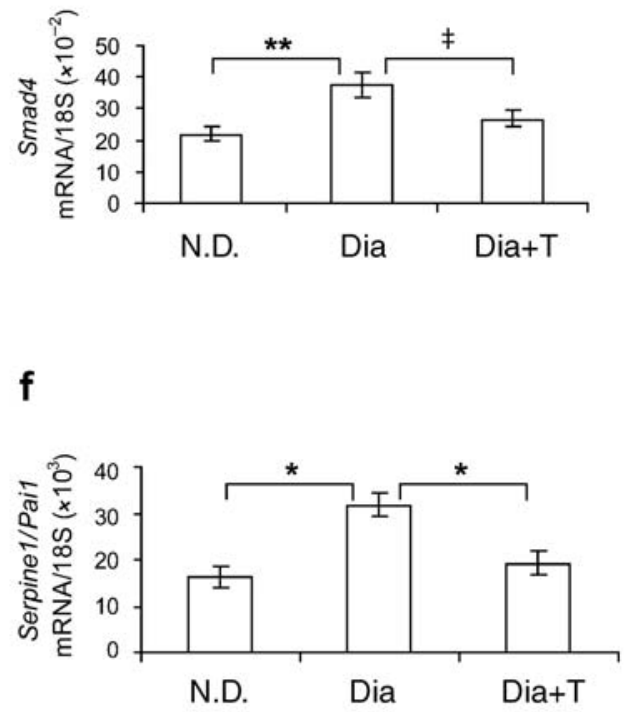

were significantly suppressed by TETA. TGF- $\beta 1$ is synthesised as a 391-amino acid precursor with little activity, which requires cleavage of its $\mathrm{NH}_{2}$-terminal latency-associated peptide to produce the active form [28]. Its activity may also be modified by the proteoglycan decorin [29] and by the scavenging protein $\alpha 2$-macroglobulin [30] such that changes in corresponding Tgfb2 mRNA expression or TGF- $\beta 1$ protein content may not accurately reflect its biological activity. In this study, therefore, we investigated the biological effects of TGF- $\beta 1$ by analysing pSMAD2, a marker of TGF- $\beta$ signalling activation [30]. Diabetes-evoked increases in renal pSMAD2 protein were attenuated by TETA (Fig. 6e). This suppression of pSMAD2 formation was well correlated with the concomitant decreases in other fibrogenic markers observed here.

TGF- $\beta 1$ is a key cytokine in the pathway underlying tissue fibrosis and can be activated by multiple upstream effectors. Elevation of its content in mesangial cells was shown to be activated by the $\mathrm{Cu}$-dependent matricellular protein, secreted protein acidic and rich in cysteine (SPARC) [31], while collagen accumulation was reportedly decreased in SPARC-null mice with pulmonary fibrosis [32]. Moreover, inhibition of TGF- $\beta$ content was associated with inhibition of lung and liver fibrosis by tetrathiomolybdatemediated $\mathrm{Cu}$-lowering [33]. However, it remains unknown whether increased renal $\mathrm{Cu}$ (Fig. 1) upregulates SPARC and 
whether TETA inhibits diabetes-induced renal fibrosis through inhibition of SPARC.

Diabetic nephropathy is characterised by relentless accumulation of ECM components. Decreased ECM degradation also plays a role in the progression of renal lesions to fibrosis [34]. Here, TETA normalised Serpine1/ Pail mRNA and its corresponding SERPINE1/PAI1 protein in diabetic renal cortex, accompanied by decreased levels of TGF- $\beta 1$ and reversal of numerous pathophysiological changes. PAI- 1 has been reported to control TGF- $\beta$ production and thereby regulate overall ECM production in diabetes [35]. Conversely, TGF- $\beta$, acting via SMAD activation, can induce expression of Serpine1/Pail, whose promoter contains SMAD-binding elements. TETA could thus interfere with the vicious cycle of reciprocal stimulation between PAI- 1 and TGF- $\beta 1$, thus ameliorating excessive ECM deposition and subsequent renal fibrosis in diabetes.

The $\mathrm{Cu}$-dependent enzyme SSAO reportedly plays an important role in ECM deposition and maintenance in vascular smooth muscle [36]. Here, SSAO protein was increased in the diabetic kidney and this increase was downregulated by TETA (ESM Fig. 1). Interestingly, our recent proteomic analysis also showed that content of SSAO in the aortas of diabetic rats was significantly increased and that TETA normalised this overproduction (D. Gong, X. Chen, A. R. J. Phillips and G. J. S. Cooper, unpublished observations). By contrast, TETA had no effect on levels of two other $\mathrm{Cu}$-containing enzymes, SOD-1 and SOD-3 (ESM Fig. 1). In the current study, total $\mathrm{Cu}$ content was increased in the diabetic kidney (Fig. 1), a finding consistent with our prior report of elevated chelatable $\mathrm{Cu}$ in the hearts of diabetic rats [6]; both of these abnormalities were ameliorated by TETA treatment. Thus, TETA could ameliorate diabetic nephropathy by inhibiting the elevated SSAO levels associated with increased $\mathrm{Cu}$ concentrations in diabetic kidneys.

One answer to the question of how renal $\mathrm{Cu}$ accumulation occurs in diabetes is that it could be due to elevated renal AGE [37]. Multiple pathways lead to AGE accumulation in tissues in diabetes and diverse AGE products are formed. AGE deposition has been implicated in animal models of diabetic nephropathy [38], and diabetes exacerbated AGE accumulation in the veins of end-stage renal failure patients [39]. Glycation reportedly increased in vitro $\mathrm{Cu}$ binding to collagen [40]. A second possibility is endothelin-1, which was reported to mediate alteration of trace metals including $\mathrm{Cu}$ in the liver and kidneys of chronically diabetic rats [41]. Both Menkes' disease and Wilson's disease are also known to cause renal $\mathrm{Cu}$ accumulation associated with renal pathology. Accumulation of $\mathrm{Cu}$ in the proximal tubules causes mildly disturbed tubular function in Menkes' disease [42, 43], a disorder caused by mutations in the Cu-transporting ATPase gene, ATP7A. Patients with Wilson's disease suffer from haematuria, renal stones and tubular dysfunction [44]. Increased renal $\mathrm{Cu}$ levels $[45,46]$ and tissue damage [47] were also observed in Long-Evans Cinnamon rats, an animal model of Wilson's disease. This increased $\mathrm{Cu}$ accumulation is due to a defect in a second Cu-transporting ATPase, ATP7B. Thus, these $\mathrm{Cu}$-induced renal pathologies appear similar to that in diabetic nephropathy, but the molecular defects that cause tissue localisation and, of relevance to the current findings, renal $\mathrm{Cu}$ accumulation in these diseases differ from that of diabetic nephropathy.

Diabetes-evoked renal $\mathrm{Cu}$ accumulation may induce expression of the metal-binding protein, metallothionein. Although metallothionein mainly acts as a regulator of metal homeostasis, it may also be an adaptive protein that protects cells from oxidative stress. The protein is highly expressed in proximal convoluted tubular cells, which are known to be the primary site of the nephrotoxicity caused by heavy metals [48]. Elevated levels of renal metallothionein-I and metallothionein-II were identified in insulin-deficient diabetic rats. The altered metabolism of renal metallothionein was largely due to accumulation of excessive dietary $\mathrm{Cu}$ in the kidney [49]. These studies suggest that metallothionein could play a renoprotective role in diabetic nephropathy.

In summary, we have shown here that TETA, a synthetic tetramine that acts as a highly selective tetradentate $\mathrm{Cu}^{\mathrm{II}}$ chelator [7, 50], attenuates diabetic kidney disease. It does this, at least in part, by suppressing activation of the TGF- $\beta$ signalling pathway, which otherwise mediates increased content of ECM proteins and consequent renal fibrosis. The TETA-mediated decrease in the $\mathrm{Cu}^{\mathrm{II}}$-dependent enzyme SSAO (ESM Fig. 1) could contribute to this effect. Our findings implicate diabetes-evoked renal $\mathrm{Cu}^{\mathrm{II}}$ build-up, probably mainly in the ECM [6], as a key early defect in the mechanism by which diabetes leads to or causes diabetic nephropathy. They also provide a molecular explanation, at least in part, for the mechanism by which TETA acts to improve renal function in diabetes.

Acknowledgements We thank P. J. Scott for his ongoing encouragement and advice, and acknowledge support from the following sources: the Endocore Research Trust; the Health Research Council of New Zealand; the Foundation for Research, Science and Technology (FRST, New Zealand); the Maurice \& Phyllis Paykel Trust; the NZ Lottery Grants Board; The University of Auckland; and Protemix Corporation. We thank C. A. Tse, H. Liu, S. FitzPatrick and V. Tintinger for technical and administrative assistance.

Duality of interest D. Gong, J. Lu, X. Chen, S. Reddy, D. J. Crossman, Y.-S. Choong, S. Zhang, Y.-K. Chan, A. R. J. Phillips and G. J. S. Cooper declare associations with Protemix Corporation, Auckland, New Zealand. All other authors declare that there is no duality of interest associated with this manuscript. 


\section{References}

1. Susztak K, Raff AC, Schiffer M, Bottinger EP (2006) Glucoseinduced reactive oxygen species cause apoptosis of podocytes and podocyte depletion at the onset of diabetic nephropathy. Diabetes $55: 225-233$

2. Roberts AB, McCune BK, Sporn MB (1992) TGF-beta: regulation of extracellular matrix. Kidney Int 41:557-559

3. Yamamoto T, Nakamura T, Noble NA (1993) Expression of transforming growth factor $\beta$ is elevated in human and experimental diabetic nephropathy. Proc Natl Acad Sci USA 90:18141818

4. Bruijn RA, Roos A, de Geus B (1994) Transforming growth factor-beta and the glomerular extracellular matrix in renal pathology. J Lab Clin Med 123:34-47

5. Haralson MA (1997) Transforming growth factor-beta, other growth factors, and the extracellular matrix. J Lab Clin Med 130:455-458

6. Cooper GJS, Phillips ARJ, Choong SY et al (2004) Regeneration of the heart in diabetes by selective copper chelation. Diabetes 53:2501-2508

7. Cooper GJS, Chan Y-K, Dissanayake AM et al (2005) Demonstration of a hyperglycemia-driven pathogenic abnormality of copper homeostasis in diabetes and its reversibility by selective chelation: quantitative comparisons between the biology of copper and eight other nutritionally essential elements in normal and diabetic individuals. Diabetes 54:1468-1476

8. Gong D, Lu J, Chen X et al (2006) Molecular changes evoked by triethylenetetramine treatment in the extracellular matrix of the heart and aorta in diabetic rats. Mol Pharmacol 70:2045-2051

9. Leblondel G, Allain P (1988) Altered element concentrations in tissues of spontaneously hypertensive rats. Biomed Pharmacother 42:121-129

10. Minakata K, Kawai K, Horio F, Takeuchi H, Suzuki O (2004) Accumulation of copper in kidney of spontaneously hypertensive rats, a preliminary study. Biofactors 22:29-31

11. Ito S, Fujita H, Narita T et al (2001) Urinary copper excretion in type 2 diabetic patients with nephropathy. Nephron 88:307-312

12. Boomsma F, van den Meiracker AH, Winkel S, Man in't Veld AJ, Schalekamp MA (1999) Circulating semicarbazide-sensitive amine oxidase is raised both in type I (insulin-dependent), type II (non-insulin-dependent) diabetes mellitus and even in childhood type I diabetes at first diagnosis. Diabetologia 42:233-237

13. Mathys KC, Ponnampalam SN, Padival S, Nagaraj RH (2002) Semicarbazide-sensitive amine oxidase in aortic smooth muscle cells mediates synthesis of a methylglyoxal-AGE: implications for vascular complications in diabetes. Biochem Biophys Res Commun 297:863-869

14. Stolen CM, Madanat R, Marti L et al (2004) Semicarbazide sensitive amine oxidase overexpression has dual consequences: insulin mimicry and diabetes-like complications. FASEB J 18: 702-704

15. Askari FK, Dick R, Mao M, Brewer GJ (2004) Tetrathiomolybdate therapy protects against concanavalin $\mathrm{A}$ and carbon tetrachloride hepatic damage in mice. Exp Biol Med 229:857-863

16. Geist J, Werner I, Eder KJ, Leutenegger CM (2007) Comparisons of tissue-specific transcription of stress response genes with whole animal endpoints of adverse effect in striped bass (Morone saxatilis) following treatment with copper and esfenvalerate. Aquatic Toxicol 85:28-39

17. Song M, Deaciuc I, Song Z et al (2008) Tetrathiomolybdate protects against bile duct ligation-induced cholestatic liver injury and fibrosis. J Pharmacol Exp Ther 325:409-416
18. Liu D, Razzaque MS, Cheng M et al (2001) The renal expression of heat shock protein 47 and collagens in acute and chronic experimental diabetes in rats. Histochem J 33:621-628

19. Wilson G, Leiter EH (1999) Streptozotocin interactions with pancreatic beta cells and the induction of insulin-dependent diabetes. Curr Top Microbiol Immunol 156:27-54

20. Bertrand M, Weber G, Schoefs B (2003) Metal determination and quantification in biological materials using particle induced X-ray emission. Trends Anal Chem 22:254-262

21. Reddy GK, Enwemeka CS (1996) A simplified method for the analysis of hydroxyproline in biological tissues. Clin Biochem 29:225-229

22. de Zeeuw D, Remuzzi G, Parving H-H et al (2004) Proteinuria, a target for renoprotection in patients with type 2 diabetic nephropathy: lessons from RENAAL. Kidney Int 65:2309-2320

23. Chen L, Wang Y, Tay YC, Harris DC (1997) Proteinuria and tubulointerstitial injury. Kidney Int 61:S60-S62

24. Saunders GK (1986) The effects of excess renal copper on kidney function in the diabetic rat. Res Commun Chem Pathol Pharmacol $52: 45-49$

25. Walshe JM (1982) Treatment of Wilson's disease with trientine (triethylene tetramine) dihydrochloride. Lancet 1:643-647

26. Kodama H, Murata Y, Itsuka T, Abe T (1997) Metabolism of administered triethylene tetramine dihydrochloride in humans. Life Sci 61:899-907

27. Ziyadeh FN (2004) Mediators of diabetic renal disease: the case for TGF- $\beta$ as the major mediator. J Am Soc Nephrol 15: S55-S57

28. O'Conner-McCourt MD, Wakefield LM (1987) Latent transforming growth factor beta in serum. J Biol Chem 262:1409014099

29. Yamaguchi Y, Mann DM, Ruoslahti E (1990) Negative regulation of transforming growth factor-beta by the proteoglycan decorin. Nature 346:281-284

30. Massagué J, Chen YG (2000) Controlling TGF-beta signalling. Genes Dev 14:627-644

31. Lane TF, Iruela-Arispe ML, Johnson RS, Sage EH (1994) SPARC is a source of copper-binding peptides that stimulate angiogenesis. J Cell Biol 125:929-943

32. Strandjord TP, Madtes DK, Weiss DJ, Sage EH (1999) Collagen accumulation is decreased in SPARC-null mice with bleomycininduced pulmonary fibrosis. Am J Physiol 277:L628-L635

33. Brewer GJ, Ullenbruch MR, Dick R, Olivarez L, Phan SH (2003) Tetrathiomolybdate therapy protects against bleomycin-induced pulmonary fibrosis in mice. J Lab Clin Med 141:210-216

34. Lee HB, Ha H (2005) Plasminogen activator inhibitor-1 and diabetic nephropathy. Nephrology 10:S11-S13

35. Rerolle JP, Vigneau C, Hertig A, Berrou J, Rondeau E (2001) Plasminogen activator inhibitor type 1: physiology and role in renal physiopathology. Nephrology 22:5-13

36. Langford SD, Trent MB, Boor PJ (2002) Semicarbazide-sensitive amine oxidase and extracellular matrix deposition by smoothmuscle cells. Cardiovasc Toxicol 2:141-150

37. Ahmed MU, Thorpe SR, Baynes JW (1996) Identification of $\mathrm{N}$-(carboxymethyl)lysine as a degradation product of fructose lysine in glycated protein. J Biol Chem 261:4889-4894

38. Williams ME (2006) New potential agents in treating diabetic kidney disease: the fourth act. Drugs 66:2287-2298

39. Nazratun N, Mahmood AA, Kuppusamy UR, Ahmad TS, Tan SY (2006) Diabetes mellitus exacerbates advanced glycation end product accumulation in the veins of end-stage renal failure patients. Vascular Med 11:245-250

40. Qian M, Liu M, Eaton JW (1998) Transition metals bind to glycated proteins forming redox active "glycochelates": implica- 
tions for the pathogenesis of certain diabetic complications. Biochem Biophys Res Commun 250:385-389

41. Cai L, Chen S, Evans T, Cherian MG, Chakrabarti S (2002) Endothelin-1-mediated alteration of metallothionein and trace metals in the liver and kidneys of chronically diabetic rats. Int $\mathrm{J}$ Exp Diabetes Res 3:193-198

42. Kodama H, Murata Y (1999) Molecular genetics and pathophysiology of Menkes' disease. Pediatr Int 41:430-435

43. Ozawa H, Kodama H, Kawaguchi H, Mochizuki T, Kobayashi M, Igarashi T (2003) Renal function in patients with Menkes' disease. Eur J Pediatr 162:51-52

44. Leu ML, Strickland GT, Gutman RA (1970) Renal function in Wilson's disease: response to penicillamine therapy. Am J Med Sci 260:381-398

45. Hayashi M, Miyane K, Senou M et al (2005) Inhibitory effects of trientine, a copper-chelating agent, on induction of DNA strand breaks in kidney cells of Long-Evans cinnamon (LEC) rats. Exp Anim 54:403-412
46. Tsujikawa K, Suzuki N, Shimaoka T et al (1994) Abnormal accumulation of copper-metallothionein in the liver and kidney of Long-Evans rats with a cinnamon-like coat color (LEC rats). Biol Pharm Bull 17:591-595

47. Sumi Y, Kawahara S, Kikuchi Y, Sawada J, Suzuki T, Suzuki KT (1993) Histochemical and immunohistochemical localization of copper, iron and metallothionein in the liver and kidney of LEC rats. Acta Histochem Cytochem 26:5-9

48. Suzuki-Kurasaki M, Okabe M, Kurasaki M (1997) Coppermetallothionein in the kidney of macular mice: a model of Menkes' disease. J Histochem Cytochem 45:1493-1501

49. Chen ML, Failla ML (1988) Metallothionein metabolism in the liver and kidney of the streptozotocin-diabetic rat. Comp Biochem Physiol B 90:439-445

50. Wichmann KA, Boyd PDW, Söhnel T, Allen GR, Phillips ARJ, Cooper GJS (2007) Characterization of dicarboxylic salts of protonated triethylenetetramine useful for the treatment of copperrelated pathologies. Cryst Growth Des 7:1844-1850 